\title{
Incidence and outcome of early post stroke seizures in a tertiary care hospital in New Delhi..... a prospective observational study
}

\author{
Maqsood Ahmad Dar', Vasundhara Aggarwal Saika', Joy Dev Mukherjee ${ }^{3}$, Mukesh Kumar $^{3}$, \\ Khushbu Goel ${ }^{3}$ \\ 'Department of Internal Medicine, GMC Anantnag \\ ${ }^{2}$ Department of Neurology, PSRI New Delhi \\ ${ }^{3}$ Department of Neurology, Max Superspeciality hospital saket New Delhi
}

\section{A B S T R A C T}

\begin{abstract}
Background: About $11 \%$ of all epilepsies and $30 \%$ of newly diagnosed seizures in those older than 60 years have been reported to follow cerebrovascular accidents. Acute symptomatic (early) seizures occur in 3-8\% of patients, more commonly in those with severe cortical strokes and intra-parenchymal hemorrhages. This study was undertaken to determine the incidence, risk factors and clinical outcome for early seizures after acute stroke.

Aims and objectives: To study the incidence, risk factors and clinical outcomes of early seizures in post stroke patients in a tertiary care hospital.

Study design: This prospective observational analytical study was conducted from -01-06-2017 to 31-01-2019 in Max Superspeciality Hospital Saket New Delhi. All consecutive patients of acute stroke fulfilling the inclusion and exclusion criteria were included in the study.

Inclusion criteria and exclusion criteria: All patients with acute ischemic or hemorrhagic stroke with age above 18 years, either male or female were included in the study. Patients with a history of seizure or epilepsy before admission, patients with SAH, venous infarct and causes other than vascular origin were excluded from the study.

Results and observation: Out of two hundred and fifty $(n=250)$ patients of acute stroke included in the study early seizures were diagnosed in 29 patients $(11.6 \%)$.

Conclusions: We concluded that the incidence of post stroke early seizures was $11.6 \%$ in our study. We found that Patients with post stroke early seizures had higher NIHSS score, low GCS score and higher modified Rankin score at the time of admission. We recommend that stroke scores like NIHSS, ICH, GCS and mRS should be applied to all the patients of acute stroke to stratify the patients with regard to their risk of developing early seizures and prognosticating the clinical outcome.

Key words: post-stroke, seizures, early, mRS, NIHSS

JMS; 2020;23(3) 18-23 DOI: https://doi.org/10.33883/jms.v23i3.752
\end{abstract}

\section{INTRODUCTION}

About $11 \%$ of all epilepsies and $30 \%$ of newly diagnosed seizures in those older than 60 years have been reported to follow cerebrovascular accidents ${ }^{1}$. Acute symptomatic (early) seizures occur in 3-8\% of patients, more commonly in those with severe cortical strokes and intra-parenchymal hemorrhages ${ }^{2}$. Late seizures occur in about $8 \%$ of patients by 10 years, but most seizures occur in 2 years after an acute Stroke ${ }^{3}$. This study was undertaken to determine the incidence, risk factors and clinical outcome for early seizures after acute stroke.

\begin{tabular}{l|l}
$|c|$ & Access this article online \\
\hline
\end{tabular}

\section{Definitions}

Stroke is defined according to the WHO's criteria as sudden onset of signs of focal or global disturbance of cerebral function lasting more than 24 hours with no apparent nonvascular cause ${ }^{4}$.

Correspondence:

Dr. Maqsood Ahmad Dar

Neurologist, Department of internal medicine, GMC Anantnag Email:drmaq30@gmail.com

How to cite this article: Dar MA, Saika V, Mukherjee J, Kumar M, Kumar M, Goel K. Incidence and outcome of early post stroke seizures in a tertiary care hospital in New Delhi.... a prospective observational study. JMS 2020;23(3):18-23

Received: 2020-05-28 Accepted: 2020-08-29 
Dar MA etal ; Incidence and outcome of early post stroke seizures in a tertiary care hospital in New Delhi

Early seizures are defined when they appear within the first 7 days after acute stroke onset ${ }^{5}$.

Aims and objectives: To study the incidence, risk factors and clinical outcomes of early seizures in post stroke patients in a tertiary care hospital.

Primary objective: To determine the risk factors of early seizures in post stroke patients.

Secondary objective: To determine the outcome in post stroke patients of early seizures using modified Rankin Scale.

\section{Material and methods}

Study population: Consecutive patients of acute stroke admitted in Max Superspeciality Hospital New Delhi, fulfilling the following inclusion and exclusion criteria were included in the study. This prospective observational analytical study was conducted from -01-06-2017 to 31-012019.

\section{Inclusion criteria}

1. Age above 18 years, either male or female.

2. All the consecutive patients with acute ischemic or hemorrhagic stroke.

\section{Exclusion criteria}

1. Patients with a history of seizure or epilepsy before admission.

2. Patients with causes other than vascular origin.

3. Patients with $\mathrm{SAH}$, venous infarct.

Data Collection: Acute stroke patients were subjected to detailed history and clinical examination. Data on vascular risk factors including age, gender, Hypertension (BP $>140 / 90 \mathrm{mmhg}$ ), Type II Diabetes, dyslipidemias, Ischemic Heart Disease, Atrial Fibrillation, were obtained. All patients underwent CT or MRI brain according to stroke protocol on admission. Stroke severity was evaluated at admission using the National Institute of Health Stroke Scale (NIHSS) and modified Rankin Scale (mRS). Seizures were classified as focal with or without impaired awareness or generalized tonic clonic seizures (GTCS) or nonconvulsive seizures (NCSE). Routine investigations including Hemogram, serum biochemistry especially serum Electrolytes, liver function test, kidney function test ,random blood sugar, $\mathrm{HbA1C}$ to rule out any metabolic abnormality which can be responsible for seizures were done. Electroencephalogram was done in all patients of stroke with seizures at the earliest possible time.

\section{Methods of measurement of outcome of interest:}

Primary outcome: Risk factors for early seizures were identified through logistic regression with seizures and no seizures as dependent variables and risk factors such as Hypertension, Type II Diabetes Mellitus, Hyperlipidemia were taken independent variables. Their odds ratio were worked out along with the $95 \%$ confidence interval and statistical significance was at $5 \%$ level

Secondary outcome: Clinical outcome were measured including mortality and disability at discharge according to Modified Rankin Score. mRS 0,1,2 was taken as nondisabling stroke and 3,4 , as disabling stroke

Statistical Methods: Incidence of early seizures were worked up as percentage of total cases of stroke that will have seizures within 7 days. Risk factors for early seizures were identified through logistic regression with seizures and no seizures as dependent variables and risk factors such as Hypertension, Type II Diabetes Mellitus, Hyperlipidaemia will be independent variables. There odds ratio was worked out along with the $95 \%$ confidence interval and statistical significance was at $5 \%$ level. Clinical outcomes such as post stroke mortality/ disability was assessed in terms of percentage of cases experiencing early seizures as compared with patients not experiencing seizures. These percentages were compared by Chi-square test. SPSS20 was used for computation.

Results and observation: Total two hundred and fifty $(\mathrm{n}=250)$ patients of acute stroke were included in the study .Two hundred twenty patients ( $88 \%$ ) had acute ischemic stroke and thirty patients $(12 \%)$ had acute hemorrhagic stroke. Two hundred and twenty one $(\mathrm{n}=221)$ patients $(88.4 \%)$ of acute stroke did not have early seizures. Early seizures were diagnosed in 29 patients (11.6\%). (Figure-1)

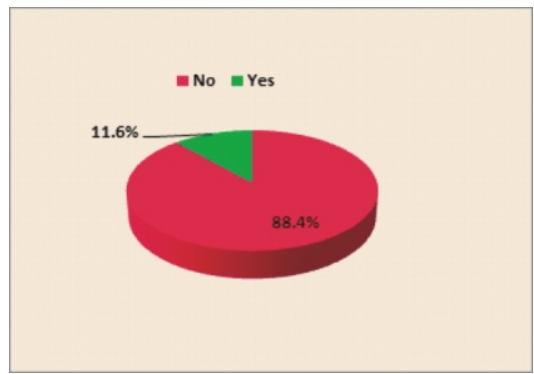

Fig. 1: Percentage post stroke early seizures 
Dar MA etal ; Incidence and outcome of early post stroke seizures in a tertiary care hospital in New Delhi

Table: 1: Demographic profile of patients with Acute stroke

\begin{tabular}{|l|l|l|l|}
\hline $\begin{array}{l}\text { Total No. of patients } \\
\text { N= 250 }\end{array}$ & \multicolumn{2}{|l|}{ Early Seizures } & \\
\hline & Yes (n= 29) & No ( $\mathbf{n = 2 2 1})$ & p Value \\
\hline $\begin{array}{l}\text { Age of patients (Mean } \pm \\
\text { SD }\end{array}$ & $64.83 \pm 16.45$ & $62.13 \pm 14.18$ & 0.396 \\
\hline Sex (M:F) & $1.4: 1$ & $2.2: 1$ & 0.272 \\
\hline $\begin{array}{l}\text { Systolic Blood Pressure } \\
\text { (mean } \pm \text { SD) }\end{array}$ & $146.50 \pm 36.62$ & $143.89 \pm 26.6$ & 0.641 \\
\hline DBP (mean \pm SD) & $82.77 \pm 18.49$ & $85.15 \pm 12.3$ & 0.508 \\
\hline $\begin{array}{l}\text { Level of Consciousness } \\
\text { GCS (mean } \pm \text { SD) }\end{array}$ & $\mathbf{8 . 4 1} \pm \mathbf{4 . 8 8}$ & $\mathbf{1 4 . 6 4} \pm \mathbf{1 . 5 0}$ & $<\mathbf{0 . 0 0 1}$ \\
\hline CAD & $7(41.7 \%)$ & $46(20.8)$ & 0.681 \\
\hline HTN & $22(75.5 \%)$ & $139(62.9 \%)$ & 0.170 \\
\hline AF & $4(7.2 \%)$ & $16(13.8 \%)$ & 0.385 \\
\hline HbA1C (mean \pm SD) & $5.94 \pm 1.15$ & $6.27 \pm 1.69$ & 0.328 \\
\hline
\end{tabular}

\section{Risk factors for acute stroke with early seizures:}

All the patients were evaluated for preexisting risk factors like glycemic control (Hbalc), presence of coronary artery disease, Type II Diabetes Mellitus, Hypertension, and ECG abnormities like atrial fibrillation. No significant difference in occurrence of ES was noticed in relation to premorbid risk factors as shown in Table 2.

Table 2: Showing co morbidities of patients included in the study.

\begin{tabular}{|c|c|c|c|c|c|}
\hline \multirow[b]{3}{*}{ HTN } & \multicolumn{4}{|c|}{ Early seizures } & \multirow[t]{3}{*}{$\mathrm{P}$ value } \\
\hline & \multicolumn{2}{|c|}{ No } & \multicolumn{2}{|c|}{ Yes } & \\
\hline & $\bar{n}$ & $\%$ & $\bar{n}$ & $\%$ & \\
\hline no & 82 & 37.1 & 7 & 24.1 & \multirow[t]{3}{*}{0.170} \\
\hline yes & 139 & 62.9 & 22 & 75.9 & \\
\hline total & 221 & 100 & 29 & 100 & \\
\hline DM & & \multirow[t]{4}{*}{0.573} \\
\hline No & 125 & 56.6 & 18 & 62.1 & \\
\hline Yes & 96 & 43.4 & 11 & 37.9 & \\
\hline Total & 221 & 100 & 29 & 100 & \\
\hline ECG & & \multirow[t]{4}{*}{0.616} \\
\hline Non AF & 203 & 91.8 & 24 & 82.7 & \\
\hline $\mathrm{AF}$ & 18 & 8.14 & 5 & 17.24 & \\
\hline Total & 221 & 100 & 29 & 100 & \\
\hline \multicolumn{5}{|l|}{ CAD } & \multirow[t]{4}{*}{0.681} \\
\hline No & 175 & 79.2 & 22 & 75.9 & \\
\hline Yes & 46 & 20.8 & 7 & 24.1 & \\
\hline Total & 221 & 100 & 29 & 100 & \\
\hline
\end{tabular}

Ischemic stroke were classified as cortical, subcortical and combined cortical and subcortical based on neuroimaging. Size of the ischemic infarcts was classified as small if lesion is $<1.5 \mathrm{cms}$, medium if lesion was in a cortical superficial branch of MCA or lesion was involving the MCA deep branch, or lesion is in a cortical superficial branch of PCA or lesion was involving the PCA branch or lesion in a cortical superficial branch of ACA. Large if the lesion was involving the whole territory of ACA or lesion was involving 2 cortical superficial branches of MCA or the lesion was involving a cortical superficial branch of MCA associated to the MCA deep branch or the lesion was involving more than 1 artery territory and lesions involving Brainstem or Cerebellum $>1.5 \mathrm{cms}$. We found that $45.6 \%$ had cortical, $29.2 \%$ had subcortical and $13.2 \%$ had combined cortical and subcortical involvement. When the area of infarct was compared in patients of acute stroke with and without early seizures, it was found that $58.8 \%$ of patients of early seizures $(n=10)$ had combined cortical and subcortical involvement which is statistically significant $(\mathrm{p}=<0.001$, Table 3 .

Table: 3: GCS score of patients with acute stroke with or without seizures at admission

\begin{tabular}{|l|l|l|l|l|l|}
\hline \multirow{2}{*}{ Infarct Regions in MRI/CT } & \multicolumn{4}{|c|}{ Early Seizure } & p \\
\cline { 2 - 6 } & \multicolumn{3}{|c|}{ value } \\
\cline { 2 - 6 } & $\mathbf{n}$ & $\mathbf{\%}$ & $\mathbf{N}=$ & $\mathbf{N}$ & \\
\hline Cortical & 112 & 55.2 & 2 & 11.8 & $<0.00$ \\
\hline Subcortical & 68 & 33.5 & 5 & 29.4 & 1 \\
\hline Cortical+ Subcortical & 23 & 11.3 & 10 & 58.8 & \\
\hline Total & $\mathbf{2 0 3}$ & 100 & 17 & 100 & \\
\hline
\end{tabular}

We found that $42.2 \%$ patients had small infarct, $39.6 \%$ had medium size infarct and $12.7 \%$ had large infarct on CT /MRI. Seventy percent $(70.5 \%)$ of patients $(n=12)$ of early seizures had large infarct on CT/MRI which is statistically significant as shown in Figure -2

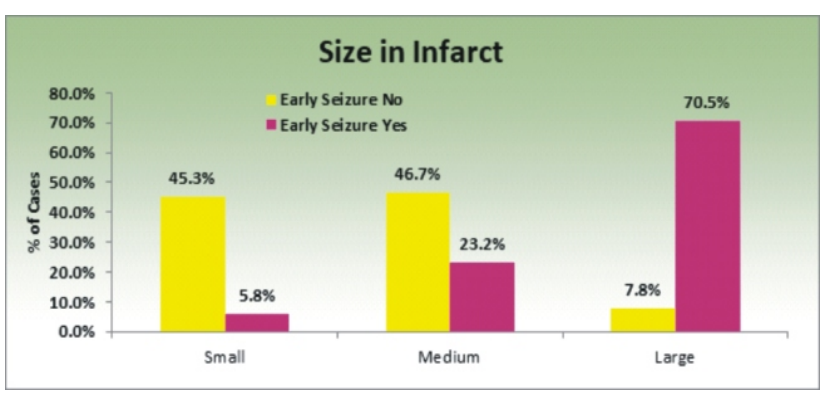

Fig. 2 :mRS score at admission in patients of acute stroke with and without ES

In patients of hemorrhagic stroke, the size was considered large if the maximal diameter of the lesion was $>3 \mathrm{cms}$. Out of 12 patients of ICH with ES, $91.6 \%(n=11)$ of patients had large bleed which is statistically significant. ICH score was evaluated in patients with hemorrhagic stroke at admission. Out of 30 patients, 12 patients ( $40 \%$ ) had ICH score of $\geq 3$. When the comparison was made between the patients with and without seizures, the occurrence of ES was significantly higher $(66.66 \%$ vs $22.2 \%)$ with ICH score of 
Dar MA etal ; Incidence and outcome of early post stroke seizures in a tertiary care hospital in New Delhi

$\geq 3 .(\mathrm{p}<0.001$.Table-4.

Table 4 : ICH score of patients with acute stroke with and without early seizure

\begin{tabular}{|l|l|l|l|l|l|}
\hline \multirow{3}{*}{ ICH score } & \multicolumn{3}{|c|}{ Early Seizure } & \multirow{2}{*}{ V } \\
\cline { 2 - 5 } & \multicolumn{3}{|c|}{ No } & Yes & \\
\cline { 2 - 5 } & $\mathbf{n}$ & $\mathbf{\%}$ & $\mathbf{n}$ & $\mathbf{\%}$ & \\
\hline$<3$ & 14 & $77.7 \%$ & 4 & $33.3 \%$ & \multirow{2}{*}{0.001} \\
\hline$\geq 3$ & 4 & $22.22 \%$ & 8 & $66.6 \%$ & \\
\hline Total & 18 & $100 \%$ & 12 & $100 \%$ & \\
\hline
\end{tabular}

The most common type of seizure observed was generalized tonic clonic seizure (41.37\%). Eleven patients (37.9\%) had non-convulsive status epileptics and 6 had focal seizures $(20.68 \%)$.

GCS score was evaluated in all patients of acute stroke at admission. It was observed that $91.6 \%$ had GCS score more than 8 and $8.4 \%$ had GCS of $\leq 8$. In patients with early seizures, $55.17 \%$ had a score $\leq 8$ and $44.8 \%$ had score more than 8 . The mean GCS score of patients with early seizures was $(8.41+4.88)$ and without seizures was $(14.64+1.50)$ which was statistically significant $(\mathrm{p}=<0.001)($ Table-5)

Table: 5: GCS score of patients with acute stroke with or without seizures at admission

\begin{tabular}{|l|l|l|l|l|l|}
\hline \multirow{2}{*}{$\begin{array}{l}\text { GCS at } \\
\text { admission }\end{array}$} & \multicolumn{4}{|c|}{ Early Seizure } & \multirow{2}{*}{ p Value } \\
\cline { 2 - 5 } & No & \multicolumn{1}{|c|}{ Yes } & \\
\cline { 2 - 5 } & $\mathrm{n}$ & $\%$ & $\mathrm{n}$ & $\mathbf{\%}$ & \\
\hline$>8$ & 216 & 97.7 & 13 & 44.8 & $<0.001$ \\
\hline$\leq 8$ & 5 & 2.26 & 16 & 55.17 & \\
\hline Total & 221 & 100 & 29 & $100 \%$ & \\
\hline
\end{tabular}

All the patients of acute stroke at admission were evaluated with NIHSS for assessing the severity of stroke. Two hundred and eleven patients $(84.4 \%)$ had score of less than 16 and $15.6 \%(\mathrm{n}=39)$ had score of $\geq 16$. When comparison was made between two groups with and without early seizures, it was found that $72.41 \%$ of patients with early seizures had a NIHSS score of more than $\geq 16$ at admission and only $8.14 \%$ of patients without seizures had the score of $\geq 16$. The mean NIHSS score in patients with early seizures at admission was (13.76 \pm 7.68$)$ and those without seizures was $(5.77 \pm 4.31)$ which was statistically significant $(\mathrm{p}<0.001)$ (Table 6$)$

Table: 6: NIHSS score in patients of acute stroke with and without seizures

\begin{tabular}{|l|l|l|l|l|l|}
\hline \multirow{2}{*}{$\begin{array}{l}\text { NIHSS at } \\
\text { admission }\end{array}$} & \multicolumn{2}{|l|}{ Early Seizure } & \multirow{2}{*}{ p Value } \\
\cline { 2 - 5 } & No & \multicolumn{1}{|l|}{ Yes } & \\
\cline { 2 - 5 } & $\mathbf{n}$ & $\mathbf{\%}$ & $\mathbf{n}$ & $\mathbf{\%}$ & \\
\hline$<16$ & 203 & 91.8 & 8 & 27.58 & $<0.001$ \\
\hline$\ldots$ & 18 & 8.14 & 21 & 72.41 & \\
\hline Total & 221 & 100 & 29 & 100 & \\
\hline
\end{tabular}

The mRS score was evaluated in all patients of acute stroke at admission. Eighty patients (32\%) had mRS score of $<2$, and 170 patients $(68 \%)$ had score of $\geq 2$ at admission. When mRS score at admission was compared between patients with early seizures and without seizures, it was found that early seizures occurred in $89.6 \%$ of patients of acute stroke with a score of $\geq 2$. These findings were statistically significant $(\mathrm{p}<0.001$ - Figure - 3$)$.

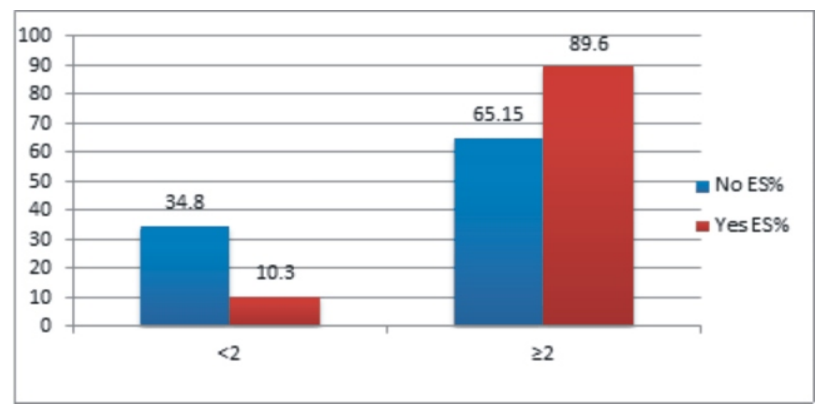

Fig. 3 : mRS score at admission in patients of acute stroke with and without ES

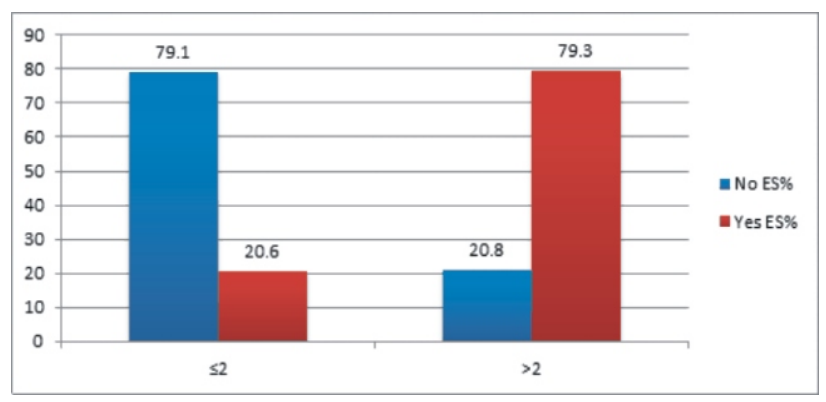

Fig. 4: mRS score at discharge of patients of acute stroke

Modified Rankin Score at discharge: Sixty nine patients had disabling stroke $\quad(\mathrm{mRS}>2)$ at discharge. Out of these ,twenty three patients (79.3\%) had ES. The mean mRS at discharge was 41.69 in patients of acute stroke with early seizures as compared to without seizures which is statistically significant $(\mathrm{p}<0.001)$ ( Table 7).

Table-7: Mean mRS score of patients with post stroke early seizures at discharge

\begin{tabular}{|c|c|c|c|c|c|c|c|}
\hline & \multicolumn{6}{|c|}{ Early Seizure } & \multirow{3}{*}{$\begin{array}{l}\text { p } \\
\text { Value }\end{array}$} \\
\hline & \multicolumn{3}{|c|}{ No } & \multicolumn{3}{|l|}{ Yes } & \\
\hline & sont & Median & $\begin{array}{l}\text { Min - } \\
\text { Max }\end{array}$ & $\begin{array}{l}\text { Mean } \pm \\
\text { SD }\end{array}$ & Median & $\begin{array}{l}\text { Min - } \\
\text { Max }\end{array}$ & \\
\hline $\begin{array}{l}\mathrm{mRS} \text { at } \\
\mathrm{D} / \mathrm{S}\end{array}$ & ${ }_{2 x t}$ & 2.00 & $1-6$ & $\begin{array}{l}4.00 \pm \\
1.69\end{array}$ & 4.00 & $1-6$ & $<0.001$ \\
\hline
\end{tabular}

Improvement or deterioration of $\mathrm{mRS}$ score at discharge :

It was observed that $31 \%$ of patients of acute stroke with early seizures had worsening of mRS score at discharge (over a period of 7 days ) as compared to only $5 \%$ of 
Dar MA etal ; Incidence and outcome of early post stroke seizures in a tertiary care hospital in New Delhi

patients without seizure group who showed worsening which is statistically significant $(\mathrm{p}=<001)$. Figure -5$)$.

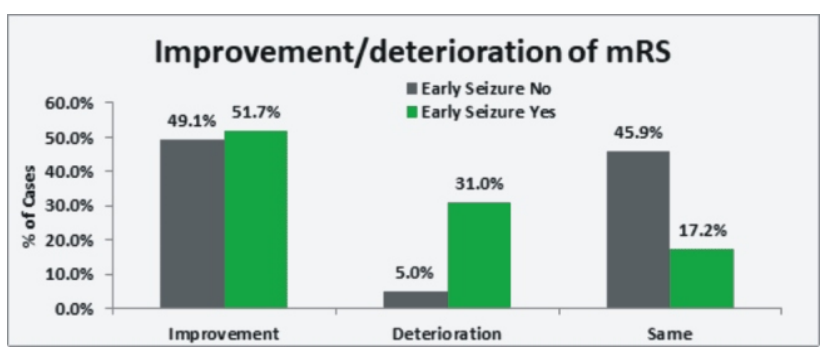

Fig. 5: Improvement and deterioration of mRS of patients with acute stroke with and without ES at discharge.

\section{DISCUSSION:}

The rate of early seizures after stroke varies widely in the literature $(2.742 .8 \%)$ due to different inclusion criteria, study designs, and detection modes6-14. The Oxford shire community stroke 15 project suggested a cumulative risk of $11.5 \%$ for the development of seizures within 5 years. The incidence of early seizures in our study was almost same about $11.6 \%$. However, the reported rate of post stroke early seizures in our study was higher than that reported $(2 \%$ to $6 \%$ ) in other prospective studies $3,2,16$. We did not find any significant association between the presence of comorbidities like Hypertension, Diabetes mellitus, Ischemic Heart disease, Atrial fibrillation with post stroke early seizures, which is consistent with studies published by Labovitz et al10 and Lamy et a 16 . We found that a higher NIHSS score $(>20)$ increases the risk for the development of seizures. This finding is supported by Bladinet al , Goswami RP et al17 and Cornado J et al7. Szaflarski et al 18 found that the patients with seizures had significantly lower initial Glasgow Coma Scale (GCS) scores, higher National Institutes of Health (NIH) Stroke Scale (NIHSS) score, higher 30-day mortality and had a higher incidence of hemorrhagic stroke than patients without seizure. It was also found that prestroke modified Rankin Score $(\mathrm{mRS})>1$ was an independent risk factor for development of post stroke seizures. Along with NIHSS score we measured the stroke severity with mRS and GCS at admission. We found that patients with post stroke early seizures had lower GCS score $(<10)$ and higher $\mathrm{mRS}(\geq 2)$ at admission in comparison with those patients without seizures. Significant number of patients with seizures had deterioration of $\mathrm{mRS}$ in the hospital leading to increase mortality and disability. So in our study low GCS score, high NIHSS and high Modified Rankin Score were independently associated with development of post stroke seizures.

\section{CONCLUSIONS:}

We made following conclusions:

1. The incidence of post stroke early seizures is $11.6 \%$ in our study.

2. No association between Age and Gender were found between patients with $\mathrm{ES}$ and without $\mathrm{ES}$.

3. We did not find any significant correlation between presence of co morbidities like Hypertension, Diabetes mellitus, Ischemic Heart disease, Atrial fibrillation with occurrence of early seizures in patients of acute stroke.

4. Patients with post stroke early seizures had higher NIHSS score, low GCS score and higher modified Rankin score at the time of admission.

\section{RECOMMENDATION:}

We recommend that stroke scores like NIHSS, ICH, GCS and mRS should be applied to all the patients of acute stroke to stratify the patients with regard to their risk of developing early seizures and prognosticating the clinical outcome. This will enable the clinicians to standardize the patient care and treatment including use of prophylactic antiepileptic medications.

\section{Ethical consideration:}

This research was carried out in accordance with the basic principles defined in ICMR ' ethical guidelines for biomedical research on human participants (2006); CDSCO' guidence on good clinical practice for clinical research India. The study was conducted only after the approval by the Scientific and Ethics committees of the hospital participating in the study.

\section{REFERENCES:}

1 Annegers JF, Hayser WA,O'BrienPC, Whisnant JP.Population based study of seizure disorders after cerebral infarction Neurology. 1996:46;350-355

2 Labovitz DL, Hauser WA, Sacco RL.Prevalence and predictors early seizure and status epilepticus after first stroke. Neurology. 2001:57;200-206

3 So EL, Annegers JF, Hauser WA, O'Brien PC, Whisnant JP . Population- based study of seizure disorders after cerebral infarction. Neurology. 1996: 46:350-355 
Dar MA etal ; Incidence and outcome of early post stroke seizures in a tertiary care hospital in New Delhi

4 (WHO)World health organization Recommendations on stroke prevention, diagnosis and therapy.Report of the WHO Task Force on stroke and other cerebrovascular disorders. Stroke.1989; 20:1407-31

5 International League Against Epilepsy Commission on Epidemiology and prognosis, guidelines for epidemiologic studies on epilepsy. Epilepsia 1993;34:592-6

6 Burneo, J.G., Fang, J., and Saposnik, G. Impact of seizures on morbidity and mortality after stroke: a Canadian multicenter cohort study. European Journal of Neurology. 2010; 17: 5258

7 Bladin CF, Alexandrov AV, Bellavance A et al.Seizures after Stroke: a prospective multicenter study. Arch Neurol. 2000;57:1617-1622

8 Berges, S., Moulin, T., Berger, E.,Tatu, L.,Sablot, D., Challier, B. et al. Seizures and epilepsy following strokes: recurrence factors. European Neurology. 2000; 43:38

9 Dodge, P.R., Richardson, E.P., and Victor, M. Recurrent convulsive seizures as a sequel to cerebral infarction: a clinical and pathological study. Brain. 1954; 77: 610646

10 Kilpatrick, C.J., Davis, S.M., Tress, B.M.,Rossiter, S.C., Hopper, J.L., and Vandendriesen, M.L. Epileptic seizures in acute stroke. Archives of Neurology. 1990; 47: 157160
11 Meyer, J.S., Charney, J.Z., Rivera, V.M., and Mathew, N.T. Cerebral embolization: prospective clinical analysis of 42 cases. Stroke. 1971; 2: 541554

12 Lancman, M.E., Golimstok, A., Norscini, J., and Granillo, R. Risk factors for developing seizures after a stroke. Epilepsia. 1993; 34: 141143

13 Misirli, H., Özge, A., Somay, G., Erdogan, N., Erkal, H., and Erenoglu, N. Seizure development after stroke.International Journal of Clinical Practice. 2006; 60: 15361541

14 Olsen, T.S., Hogenhaven, H., and Thage, O. Epilepsy after stroke. Neurology. 1987; 37: 12091211

15 Burn J, Dennis M ,Bamford J,Sandercock P, Wade D, War low C. Epileptic seizures after a first stroke :The Oxfordshire Community Stroke Project. BMJ. 1997;315: 1582-1587

16 Lamy C, Domigo V, semah F. Early and late seizures after cryptogenic stroke in young adults. Neurology.2003:60: 400-404

17 Goswami RP1, Karmakar PS, GhoshA.Early seizures in first-ever acute stroke patients in India: incidence, predictive factors and impact on early outcome.Eur JNeurol. 2012 Oct; 19(10): 1361-6.

18 Szaflarski, J.P., Rackley, A.Y. Kleindorfer, D.O., Khoury, J., Woo, D., Miller, R. et al. Incidence of seizures in the acute phase of stroke: a population based study. Epilepsia. 2008; 49: 974981. 